\title{
Design of an efficient mobile measurement system for urban pollution monitoring
}

\author{
Andrea Bernieri, Domenico Capriglione, Luigi Ferrigno, Marco Laracca \\ DIEl, Università di Cassino e del Lazio Meridionale, Via G. Di Biasio 43, 03043, Cassino (FR), Italy
}

In recent years, the pollution monitoring in urban areas has become one of the most critical issues for local public authorities, which wish (or must) verify that pollution levels not exceed limits considered unsafe or that are regulated by local laws. Generally, the pollution monitoring is performed by using measurement stations located in few points of the region of interest since these stations are generally characterized by high costs, weights, and dimensions. Then, the pollution levels over the remaining area are predicted by means of suitable interpolation models. Due to the great variety of urban scenarios, it becomes very difficult to obtain reliable pollution levels in area in which the measurements has not been directly taken but only predicted. Consequently, the pollution monitoring can suffer of a lack of reliable information indispensable for the actuation of proper environmental management policies. In this framework, this paper proposes a mobile measurement system for the real time monitoring of environmental pollutions over urban areas. The proposed approach is based on the use of a set of vehicles, typically employed for public transportation inside the urban area, equipped with the proposed mobile measurement system allowing it to measure, store, and transmit the acquired data to a remote supervisor unit somewhere on the path followed by the vehicles. Particular attention has been paid on the definition of the metrological characteristics of the measurement devices with the aims of complying with the applicable European Directives accuracy requirements and of selecting a suitable trade-off between accuracy and cost. The experimental measurement campaign performed on a suitable urban scenario has confirmed the goodness of the proposed system.

Keywords: Pollution Monitoring; Mobile Sensor Networks; Wireless Sensor Network; Distributed Measurement Systems.

Citation: Andrea Bernieri, Domenico Capriglione, Luigi Ferrigno, Marco Laracca, Design of an efficient mobile measurement system for urban pollution monitoring, Acta IMEKO, vol. 1, no. 1, article 15, July 2012, identifier: IMEKO-ACTA-01(2012)-01-15

Editor: Pedro Ramos, Instituto de Telecomunicações and Instituto Superior Técnico/Universidade Técnica de Lisboa, Portugal

Received January $16^{\text {th }}, 2012$; In final form May $28^{\text {th }}, 2012$; Published July 2012

Copyright: (C) 2012 IMEKO. This is an open-access article distributed under the terms of the Creative Commons Attribution 3.0 License, which permits unrestricted use, distribution, and reproduction in any medium, provided the original author and source are credited

Funding: This work was supported by the Emilia Romagna Region (ITALY), call PRRIITT 2008, Measure 3.1, Action A and 3DInformaticaTM Ltd.

Corresponding author: Luigi Ferrigno, e-mail: ferrigno@unicas.it

\section{INTRODUCTION}

The timely and reliable knowledge of environmental pollution in urban areas has become, in recent years, one of the most critical issues for local public authorities, even for the not negligible impact with regard to political and social choices to be made. In particular, many local authorities wish (or must) to offer continuous services about the determination of the pollution levels, especially when they exceed limits that are considered unsafe or that are regulated by local laws [1].

In this scenario, the ability to have data timely, reliable, and well spread over the territory on which base suitable management policies for the urban community, can enable to develop several actions for safeguarding the health of the population, for optimizing the resource, and for controlling the industrial and anthropogenic emissions.

The typical set-up adopted to retrieve information on air quality is based on fixed measurement units, positioned in suitable points of the area under test. These measurement units are characterized by good metrological performance somewhat counterbalanced by high costs, weights, dimensions, large measurement times, and power consumption requirements. Values of the pollutants in different and neighboring areas are then obtained by applying suitable predicting mathematical models. The position of these units must be appropriately designed to enable a reliable extension of the information collected throughout the range of interest. However, in cases where the hilly terrain or urban structure does not allow an extension of information to wide areas of territory, additional monitoring stations should be employed. Typically, this circumstance leads to a meaningful increase of the monitoring cost that sometimes becomes prohibitive for the interested authority.

If there is a need for accurate measurements in areas where fixed stations are not installed, some innovative solutions, present in the literature, suggest the use of mobile (i.e. portable) measurement systems that, indeed, re-propose the use of fixed 
units made transportable through their installation on trucks or cars [2]-[4]. Thus, measurement campaigns can be implemented in wider parts of the territory, but the simultaneity of the data recorded is closely related to the total number of systems used for surveying. In this context, it thus becomes of interest to have mobile (i.e. moving) analysis systems that measures the environmental pollution in wider parts of a territory according to defined paths of the vehicles on which they are installed.

In this way, the cost of urban pollution monitoring can be substantially reduced avoiding the use of many more expensive fixed or transportable monitoring units. By adopting a suitable data management technique, it is then possible to perform the integration of the information in "clusters" of data. This approach makes possible, with a small number of devices, to achieve full coverage, accurate, and timely provision of pollutions in wide areas. To these aims, some measurement architectures have been presented in the literature [5]-[7]. However, they does not fully consider the issue of the reliability of data collected from the standpoint of measurement uncertainty and of the maintenance of the metrological characteristics of the units during the whole operative cycle. Then some troubles about the reliability of such solutions arise.

Thanks to previous experience in the field of wireless measurement systems and sensing devices [8], wireless sensor networks [9] and uncertainty estimation [10], in this paper the authors propose an effective mobile measurement system for the real time monitoring of environmental pollutions over urban areas [11]. Key features of the proposed system, thought to be located on public urban ground transportation vehicles, are the significant low cost, the high portability of the measurement units, the autonomous power supply, and the measurement strategy able to minimize the measurement uncertainty. To these aims, suitable interpolation techniques aggregating data acquired over the spatial and time domains can be applied.

In the following, starting from requirements of directives and laws operating in the European countries, the metrological specifications for the sensing devices are defined. Then, the architecture of the proposed measurement system is presented, together with some details about the measurement unit, the considered sensors, and the software architecture. Finally, to validate the proposed system, a measurement campaign has been performed on a suitable urban area.

\section{EUROPEAN REGULATION FOR THE AIR POLLUTANT}

This section summarizes the legal regulations and limit values for the air pollution parameters which have been considered to drive the selection of the transducers and the design of the measurement system.

In Europe, European Directive number 30 of the April 22-th 1999 related to environmental air quality limit values regulates the presence of air pollutions [12]. The considered quantities are the sulphur dioxide $\left(\mathrm{SO}_{2}\right)$, nitrogen dioxide $\left(\mathrm{NO}_{2}\right)$, nitrogen oxides $(\mathrm{NO})$, particulate matter (PM) and lead. In $2000 / 69 / \mathrm{EC}$ the ambient air quality limit values for benzene $\left(\mathrm{C}_{6} \mathrm{H}_{6}\right)$ and carbon monoxide $(\mathrm{CO})$ are given [13]. These directives are then to be acknowledged by the state trough suitable decrees. As an example, in Italy the Italian Ministerial decree number 60 of the 02/04/2002 has acknowledged these directives.

Typically, standards and laws express the limits of the

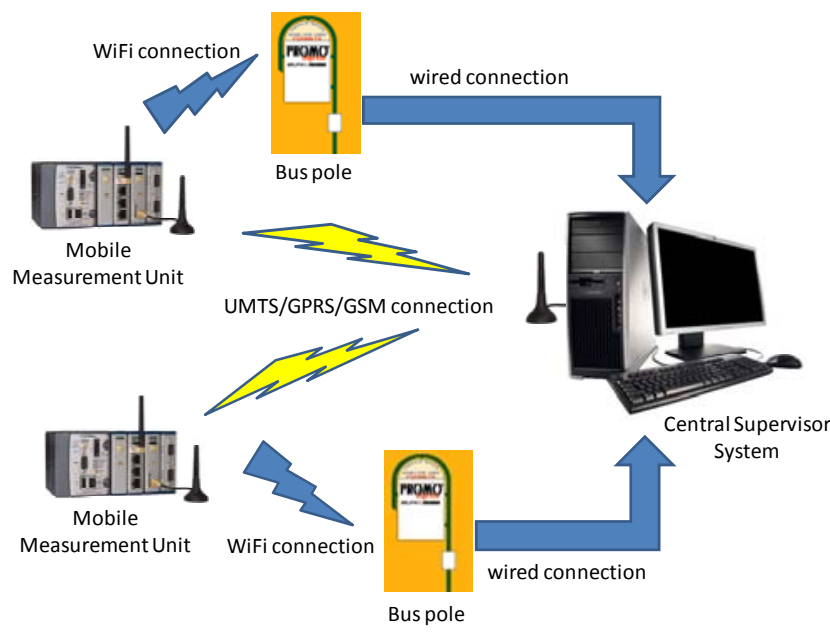

Figure 1. General architecture of the measurement system.

pollutants to which the human body can be exposed in milligram on cubic meter $\left(\mathrm{mg} / \mathrm{m}^{3}\right)$ or microgram on cubic meter $\left(\mu \mathrm{g} / \mathrm{m}^{3}\right)$, and impose limitations about the period of exposure. Table 1 summarizes these limits for the main air pollutants, and reports them also in parts per million ( $\mathrm{ppm}$ ) or parts per billion (ppb), quantities often used in the transducer specifications.

The limits reported in the table are generally referred to a mean value in a considered exposure period; on the contrary, alert thresholds are usually given in terms of maximum number of events in which the specific pollutant may exceed a corresponding limit, in a defined time period.

In the table, for the unit conversion, a reference pressure equal to $101325 \mathrm{~Pa}$ and a reference temperature equal to 298.16 $\mathrm{K}$ were considered.

\section{THE MEASUREMENT SYSTEM}

The general architecture of the proposed system is sketched in Figure 1. It is based on two main classes of devices:

- Mobile Measurement Unit (MMU): designed to be hosted on board of vehicles, as urban service buses or service vehicles of the local authority.

- Central Supervisor System (CSS): designed to be located in a fixed position and devoted to remotely access to measurement data.

The MMUs, whose architecture and components are described in detail in section 4, perform the acquisition, the collection, and the transmission of the measured data (environmental quantities and geo-localization information) to the CSS.

Table 1. Pollutant limits according to the European directives.

\begin{tabular}{cccc}
\hline Pollutant & Limit $\left[\mathrm{mg} / \mathrm{m}^{3}\right]$ & Limit [ppm] & Exposure period \\
\hline $\mathrm{CO}$ & 10 & 8.7 & daily \\
$\mathrm{NO}_{2}$ & 0.2 & 0.11 & hourly \\
& 0.03 & 0.016 & annual \\
& 0.4 & 0.21 & alert threshold \\
\hline & 0.35 & 0.13 & hourly \\
$\mathrm{SO}_{2}$ & 0.125 & 0.048 & daily \\
& 0.02 & 0.008 & annual \\
& 0.5 & 0.19 & alert threshold \\
\hline $\mathrm{O}_{3}$ & 0.18 & 0.084 & hourly \\
$\mathrm{C}_{6} \mathrm{H}_{6}$ & 0.005 & 0.144 & daily \\
\hline
\end{tabular}




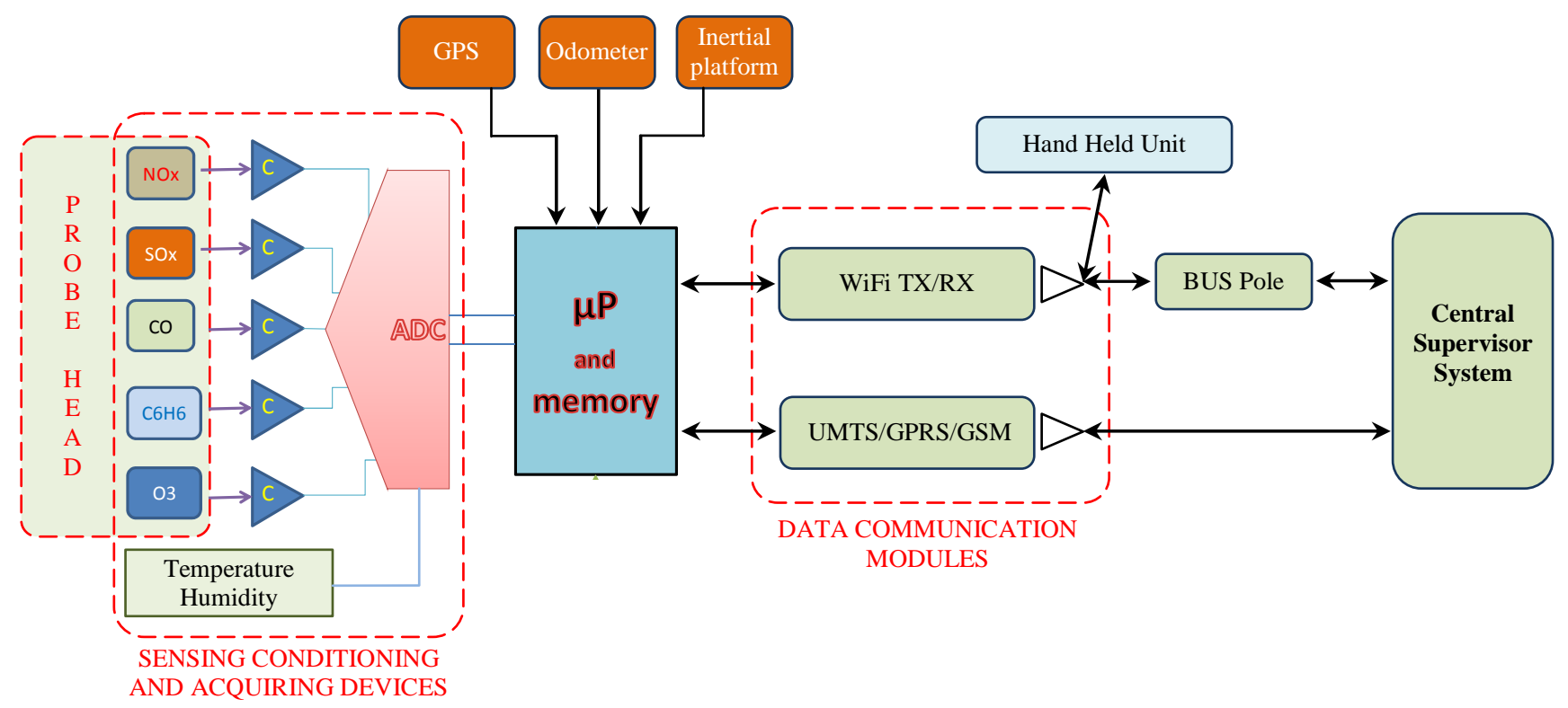

Figure 2. Architecture of the proposed Mobile Measurement Unit.

The CSS is a workstation equipped with the necessary devices and software for retrieving, storing, and viewing measurement data collected by MMUs as well as for managing the data elaboration. In particular, using appropriate software modules suitably developed, the CSS ensures:

- the management of involved MMUs by means of a graphical I/O user interface for enabling/disabling, control, configuring and verifying the MMU operation;

- the representation of each MMU position and its measurement results on a digital cartography;

- the storage of the data collected in time series for their statistical analysis over time;

- the dynamic integration of data collected from different MMUs, with reference to mathematical models of pollution diffusion in the monitored area, and data fusion techniques to correlate data relating to the same location and detected by different MMUs;

- the availability of the data processed on the Internet for a free use or a controlled use by the operators enabled.

\section{THE MOBILE MEASUREMENT UNIT}

The core of the proposed system is the Mobile Measurement Unit (MMU). It is designed and realized according to a modular architecture, in which various sensors can be combined as required by the specific needs of the measurement purposes. The sensor assembly is also designed to be easily removed from the rest of the unit, in order to perform the check and periodic maintenance necessary to ensure proper traceability and accuracy of the measurements.

With reference to Figure 2, the MMU is composed by four main sub-modules: (i) the probe head, (ii) the sensing, conditioning and acquiring devices, (iii) the processing and memory unit, and (iv) the data communication modules.

Figure 3 shows a picture of the realized MMU.

\subsection{The probe head}

A schematic of this device is reported in Figure 4. It is composed by a package in which the sensing elements are suitably placed, and by a controlled air aspiration system that is able to guarantee the desired air flux whatever the vehicle velocity is. In particular, the sampling air port is placed in the opposite direction with respect to the vehicle direction, and the air flow is fixed by the aspiration system. Inside the probe head, some fins able to impose a suitable air velocity for each considered sensing element are also placed. This choice allows the reliability of the mobile measurements to be improved.

\subsection{The sensing, conditioning, and acquiring devices}

The sensing and conditioning circuits are developed to measure the following quantities: air temperature, air humidity, and the concentrations of: Carbon monoxide (CO), Nitrogen dioxide $\left(\mathrm{NO}_{2}\right)$, Sulphur dioxide $\left(\mathrm{SO}_{2}\right)$, Ozone $\left(\mathrm{O}_{3}\right)$, and Benzene $\left(\mathrm{C}_{6} \mathrm{H}_{6}\right)$. In the future version of the measurement unit

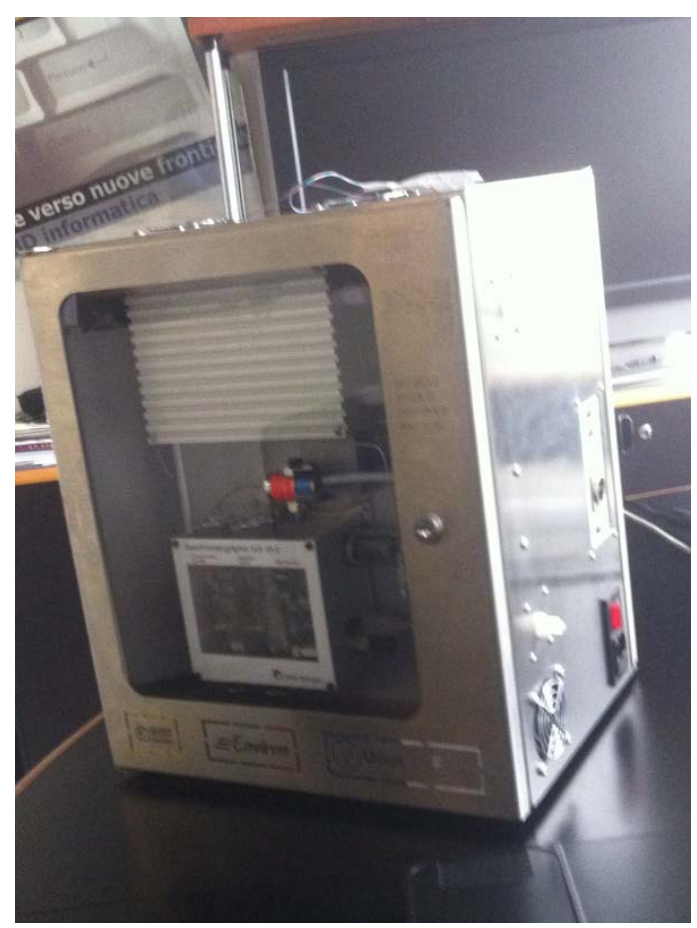

Figure 3. The realized Mobile Measurement Unit (MMU). 
also the fine particles (PMx) and other quantities of chemical interest will be considered.

The sensors are selected mainly on the basis of range, accuracy, and measurement rate, according to requirements imposed by the European directives and limits showed in the Table 1. In particular, for the pollutant quantities, Kanomax ${ }^{\mathrm{TM}}$ Aeroqual High Spec SM50 sensors are used [14]. Table 2 shows the main metrological characteristics of the selected sensors.

All sensors have an internal resistance used to bring the conversion head to a temperature that avoids any influence from external environment factors, such as atmospheric temperature and humidity, at the time of the readings. Moreover, they have the current output, typically with a load resistance ranging from 100 to $200 \Omega$, with the aim of falling in the voltage input range $0-5 \mathrm{~V}$ of the analog to digital converter. A suitable on-board Kanomax Aeroqual ADC with 0-5V input range / 12 bit resolution is adopted; in addition this converter also has an output interface based on RS232/RS485 standards.

\subsection{The processing and memory unit}

It is composed by a microprocessor and data storage hardware. The Advantech ARK 1388 industrial PC was used for the purpose. It is characterized by small dimensions and consumptions and by high robustness and good I/O capabilities [15]. The microprocessor manages the local unit and controls all the measurement and data acquisition tasks. The data are geo-referenced by means of a high-resolution GPS, embedded on the industrial PC, together with an odometer Corrsys-Datron L-400 and an inertial platform Landmark Gladiator LMRK10, which allow retrieving the geographic position even in areas not covered by GPS signal. The system also provides the local data storage necessary to save measured data between two successive data transmissions to CSS.

\subsection{The data communication modules}

The MMU has been equipped with suitable wireless mobile communication modules. In particular, $\mathrm{WiFi}^{\mathrm{TM}} \mathrm{TX} / \mathrm{RX}$ system and UMTS/GPRS/GSM modem, both embedded on the industrial PC, are used. In this way, the measured data can be transmitted to the CSS in two alternative ways: a) by means of short-range $\mathrm{WiFi}$ connections installed at the predefined locations (e.g. the bus poles); in this case the connection between the bus pole and the CSS is performed by a wired LAN connection; b) by means of long-range UMTS/GPRS/ GSM wireless connection.

The solution a) is a reliable and free of charge connection that guarantees high data rates but, due to the short range of the communication link, it is usable only at the predetermined

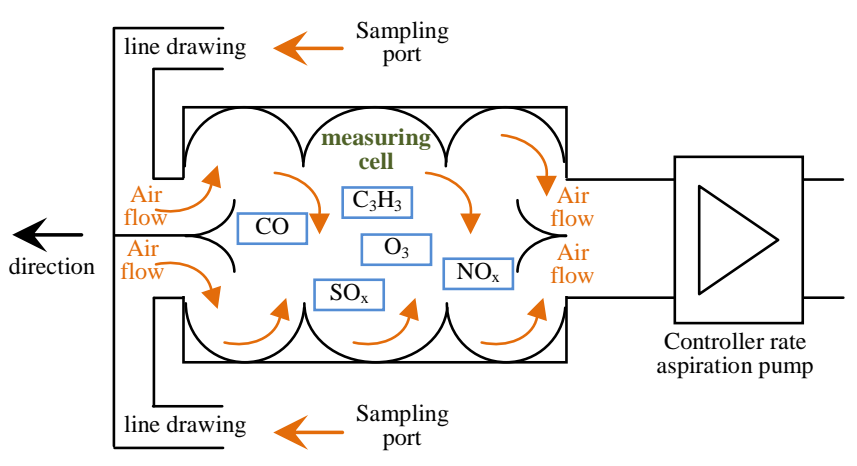

Figure 4 . Sketch of the realized probe head. locations (bus poles). Even if the measurement data are collected in real time by MMUs, they are retrievable (by the CSS) only off-line, i.e. when the vehicle is close to the bus pole. Consequently, a time delay occurs in the management of the pollution data and of the measurement system, even if it can be usually acceptable for pollution monitoring purposes.

The solution b) performs an on-line communication on long-range distance (if there is an adequate cellular network coverage), but it is generally characterized by a low data rate and not free of charge connection. Then, it is used only if the data must be on-line transmitted (e.g. alarms) or if the solution a) is temporarily out-of-order.

\section{THE SOFTWARE ARCHITECTURE}

The measurement and control software has been developed in LabView ${ }^{\mathrm{TM}}$ environment. The software is based on a clientserver architecture and it is constituted by different modules running on different devices. In particular, two main software units can be identified: i) the program running on the MMU (which operates as "measurement server"), and ii) the program running on the external remote devices (hand-held units and bus pole units which act as clients).

Figure 5 shows the general architecture of the developed software, also considering the WiFi connection of the MMU at the bus pole. As for the measurement server, all functions (data acquisition from sensors and external devices, storing and transmission) have been implemented into the firmware of the embedded PC on the MMU. Once the device has been powered on, they go in a standby mode waiting for configuration (by means of a control software running on the hand held devices) and/or for download of the storage data (by means of a download software running on the bus poles).

After the configuration phase, the whole operating is completely automatic. The firmware acquires the data from transducers and from the devices devoted to the geo localization (GPS, odometer, and inertial platform). These data are stored on the internal memory unit (as suitable files). In addition, the firmware manages the connection and the communication with the client devices. During the acquisition phase, a suitable software module allows the on-line upload of the stored data on the client devices.

As for the clients, two different software have been developed. The first one is the control software (usually running on the hand held units) that allows the connection to the MMU and, depending on the MMU's status, sends the configuration and/or start command (if the MMU is in standby mode) and, when required, the stop command (if the MMU is in acquisition mode). The second one is the download software (running on suitable unit placed on bus poles) that continuously scans the WiFi network to connect with the MMU, and then downloads all data previously stored in the MMU internal memory. It manages the data download in both the MMU standby and acquisition modes.

\section{STRATEGIES FOR POLLUTION DATA EVALUATION}

To provide a satisfying characterization of the environmental pollution, it is possible to adopt several approaches for the data sampling and interpolating over a regional scale [16], [17].

Generally, a regional distribution of the polluting can be achieved by means of either predictive models (which are characterized by a good spatial coverage and poor accuracy) or careful measurements suitably post-processed with 
Table 2. Main metrological characteristics of the selected sensors (LDL: Lower Detection Limit).

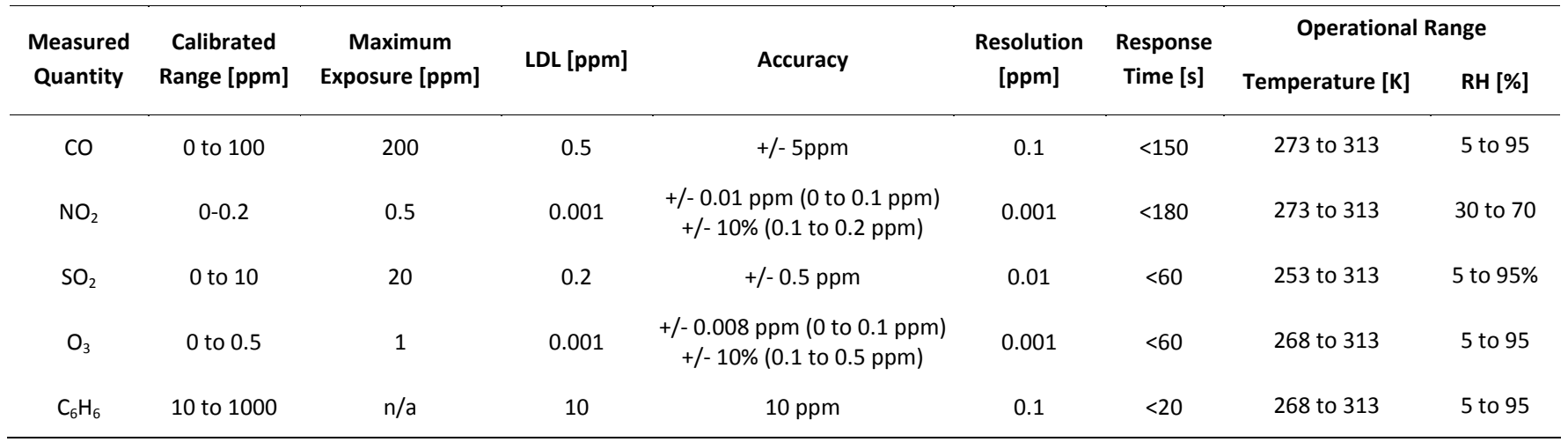

interpolation methodologies.

In the literature, we can find a number of interpolation methods developed for different applications [17], [18]. Starting from punctual data set, their aim is to obtain spatial polluting concentration fields.

These methods can be classified in two main categories, namely deterministic and geo-statistical. The main difference between these methods is that the former are only based on punctual measurements, whereas the latter exploit the data statistical spatial structure providing also the estimate of the variance on the interpolated values.

More specifically, the methods belonging to these categories are:

(a) Deterministic: classic interpolation methods such as Voronoi diagrams, Thiessen polygons, Delaunay triangles, Inverse Distance Weighting (IDW), Radial Basis Functions (RBF), Bier. (b) Geo-statistical: interpolation methods, based on the knowledge of the statistical structure of the aleatory field of the point of interest, such as the optimum interpolation, the kriging (in the various existing versions), the geo-statistical multivariate model.

In the following, a deterministic method, namely the Voronoi diagram, and a geo-statistical method, namely the ordinary kriging, were used to present the achieved results obtained by means of the proposed measurement system.

\section{EXPERIMENTAL RESULTS}

In order to evaluate the performance of the proposed measurement system, a number of measurement sessions were carried out.

For the sake of brevity, only the results related to the CO, $\mathrm{NO}_{2}$, and $\mathrm{O}_{3}$ will be reported in the following.

The measurement sessions have the following aims:

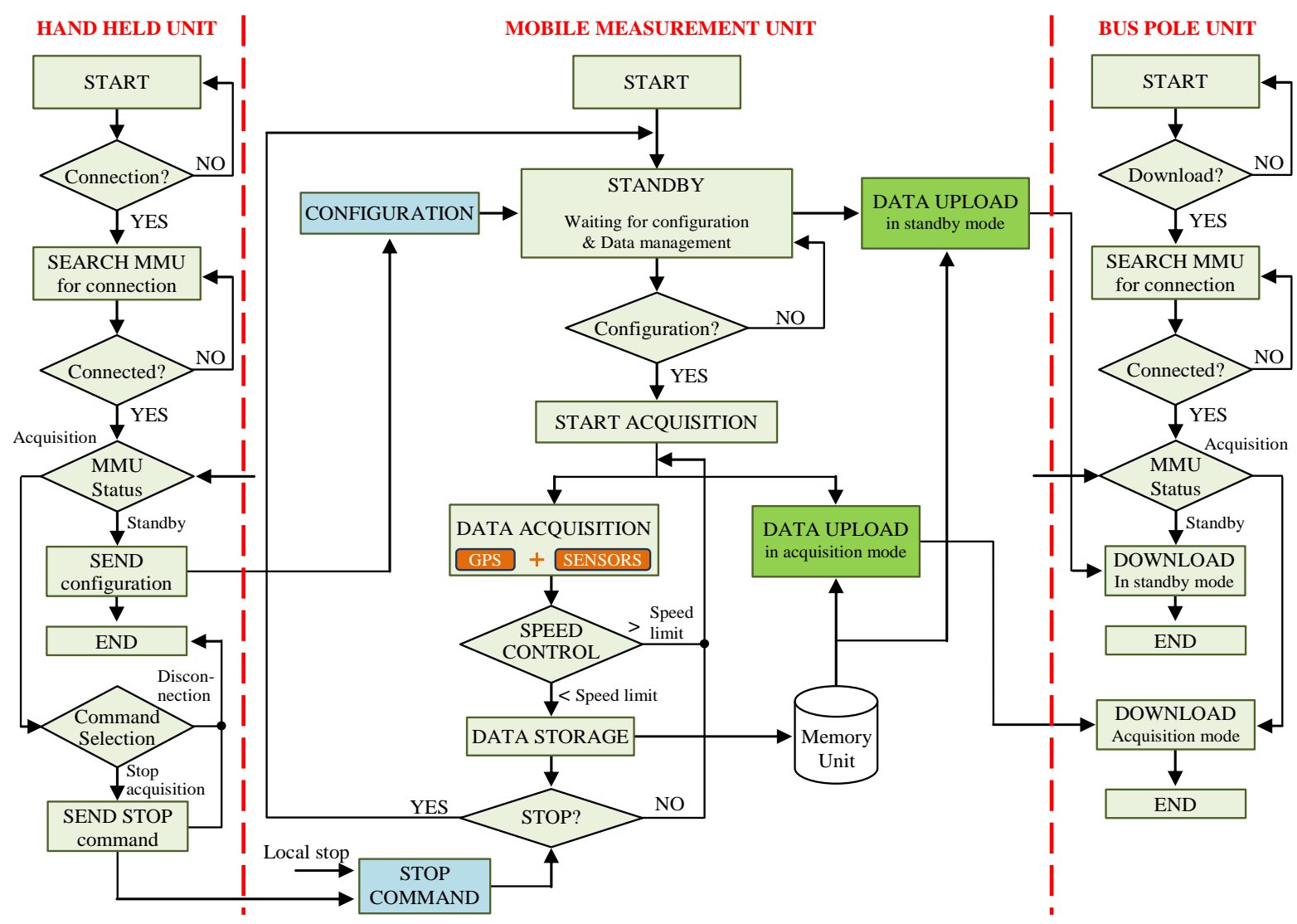

Figure 5. The architecture of the developed software. 
(i) of validating the MMU performance by means of a comparison between the results obtained from the MMU and those obtained from a calibrated traditional measurement instrumentation, positioned at the same location; to this aim, a DASIBI 2108 Chemiluminescent Nitrogen Oxide Analyzer, a DASIBI 2003 Ozone Analyzer, and a ML9830B Carbon Monoxide Analyzer were used as reference instruments;

(ii) of evaluating the distribution of pollutants in an extended area, by means of a great number of measurements acquired during predefined paths performed by using a service vehicle on which the MMU was installed; the measurements were then elaborated by means of suitable procedures, running on the CSS, which perform the elaboration of Voronoi diagrams and the kriging variograms.

To simplify this preliminary evaluation phase, the on-line data transmission from the MMU and the CSS was not performed, and the data collected during the considered paths were locally stored on the MMU and then downloaded to CSS at the end of each path.

The measurements were collected on suitable paths in the urban area of the city of Bologna, Italy, using a commercial vehicle.

For the considered pollutants, Figure 6 shows the results obtained in the (i) tests, performed during a period of about 60 hours. The results show a good agreement between the reference values and those obtained by means of the MMU, with a mean deviation always less than $10 \%$. These results are in agreement with the metrological performances of the employed sensors.

Figure 7 and Figure 8 show the considered pollutant distribution in the urban area using Voronoi diagrams and kriging variograms, respectively. In both figures, the circles indicate the punctual measurement data acquired by means of the MMU and geo-referenced using the Google map of the city. The results showed are computed as mean of seven different paths, performed in a period of one week of stable weather conditions (absence of wind and of rain). Also the single-day and the single-path results are available, but they do not show substantial differences.

In the Figures 7 and 8 , the red triangles indicate the positions of two traditional fixed measurement units. It is evident that the use of the proposed mobile measurement system is capable to highlight some urban areas in which the pollutant concentration is very high. Using the traditional approach, in these areas the pollution information may be neglected or obtained by means of prediction models which could not assure the same accuracy of the punctual measurements.

\section{CONCLUSIONS}

The paper proposes an efficient architecture for mobile systems involved in urban pollution monitoring. It is constituted by two main classes of devices: a mobile measurement unit (MMU) and remote nodes which in turn can be used both for control the MMU (and then the measurement settings) and download data collected by the MMU. The mobile measurement unit is made to be installed on public vehicles and is able to perform pollution monitoring during the usual vehicle service. The proposed measurement system is thought to provide the collected data to a suitable central supervision system which controls many measurement units and use the
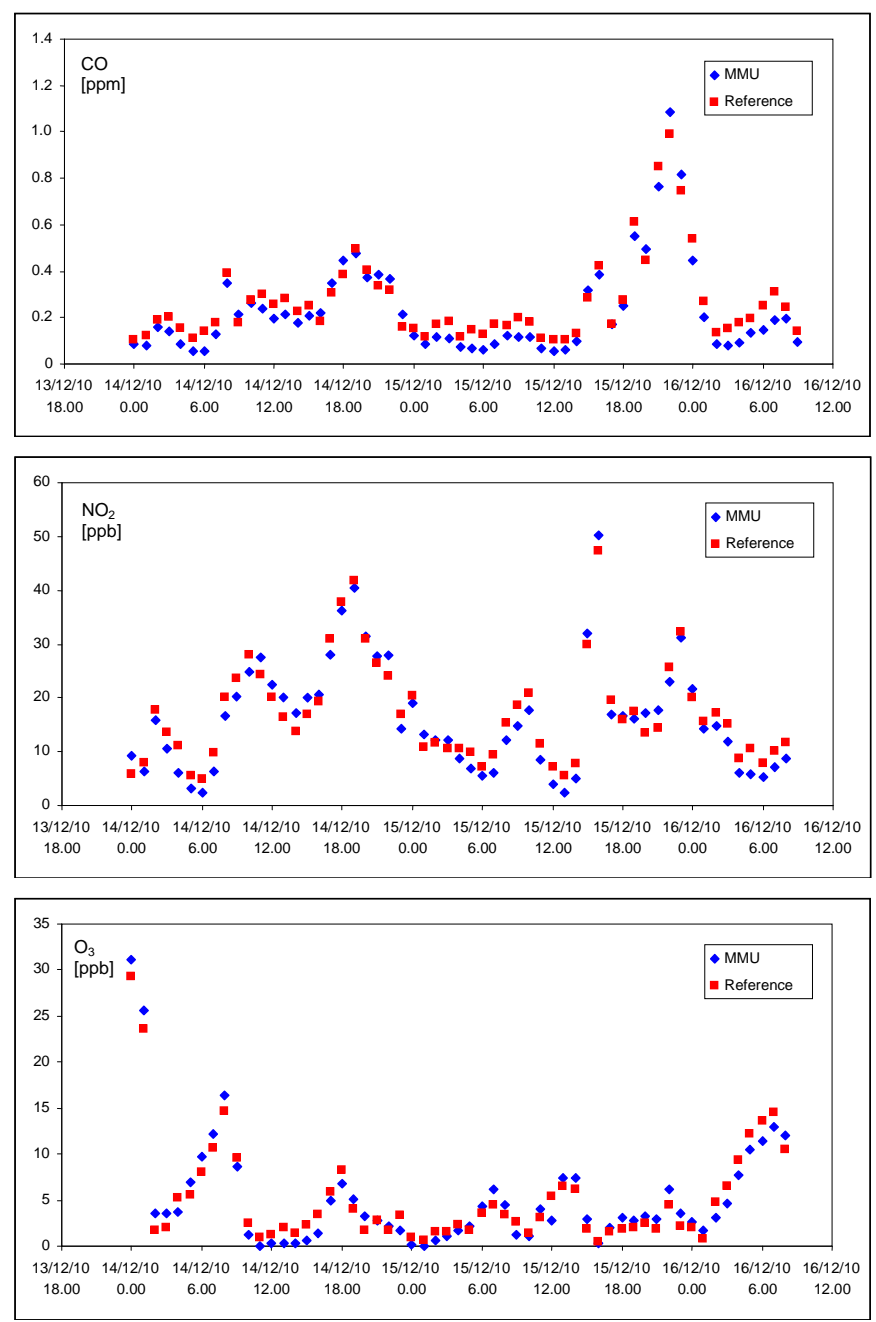

Figure 6. MMU validation test results.

acquired data to perform a spatial pollution evaluation in the area of interest (as an example by means of suitable data fusion and/or interpolation techniques).

The modular architecture of the proposed measurement system assures good feasibility and efficiency. In addition, the described solution is very attractive because allows exploiting the spatial and time capillarity of a typical public transportation system to achieve in an easy way a high amount of data (from the spatial and time points of view) useful also for improving the prediction models.

With reference to the employed data elaboration techniques (Voronoi diagrams and ordinary kriging variograms), the achieved results show a good agreement thanks to the high number of the punctual measurements carried out. However, further experimental activity has to be addressed in order to identify the best interpolation strategy related to the number and distribution of measured data and to the accuracy to be achieved.

Moreover, the use of many mobile measurement systems installed on different vehicles, which perform the same or different urban paths, can improve the whole measurement uncertainty using suitable data fusion techniques. This is the goal of the future research development, when many mobile measurement systems will be available. 

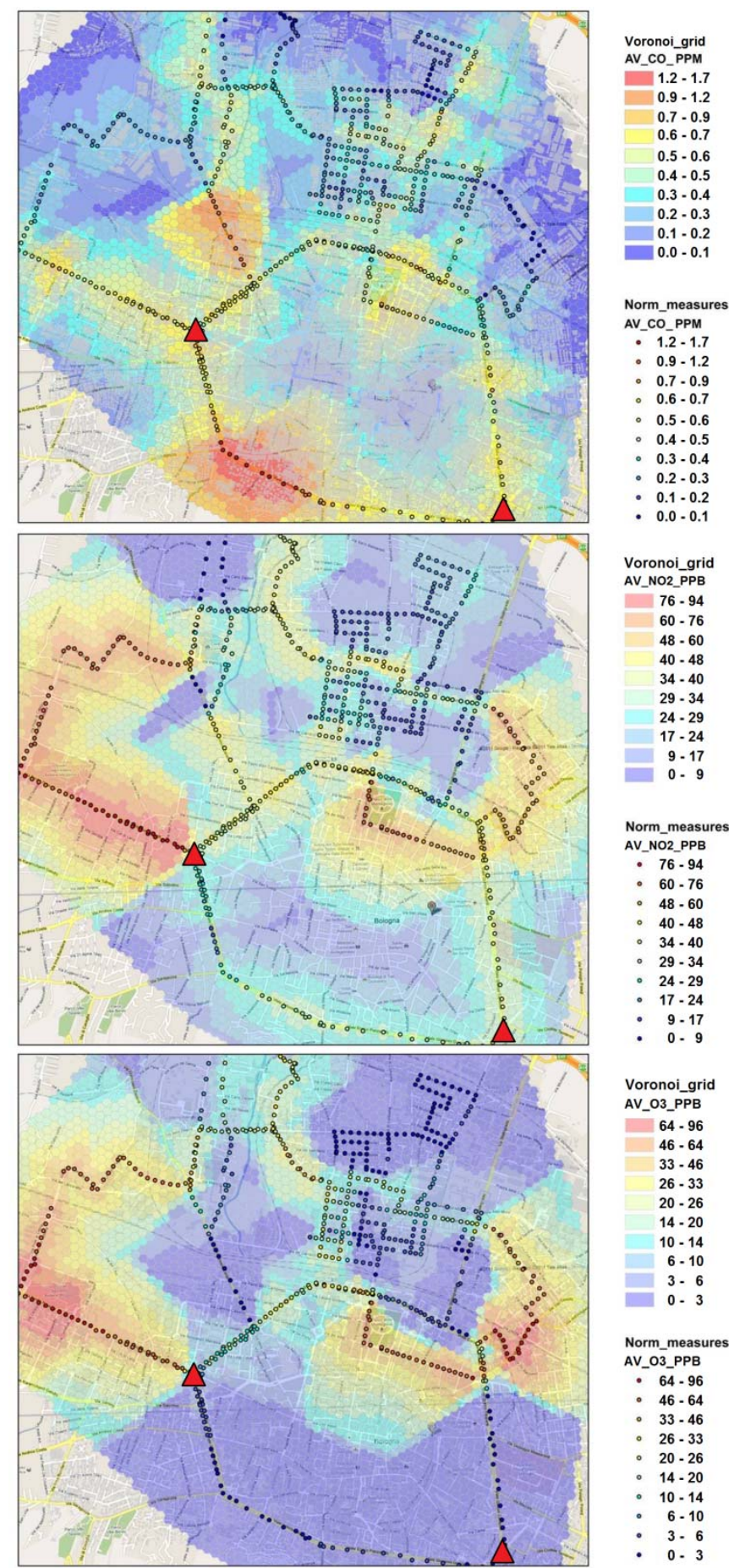

Figure 7. Voronoi diagrams of the considered pollutants (the red triangles indicate the position of traditional fixed measurement systems).

\section{REFERENCES}

[1] *** "Health Aspects of Air Pollution with Particulate Matter, Ozone and Nitrogen Dioxide", Report on a WHO Working Group, Bonn, Germany, 2003.

[2] K. D. Zoysa and C. Keppitiyagama, "Busnet - a sensor network built over a public transport system", Proceedings of the 4th European conference on Wireless Sensor Networks, 2007.

[3] D. T. N. R. Group, "Dtn reference implementation v2.3.0." 2006. [Online]. http://www.dtnrg.org/docs/ code/DTN2

[4] F. Gil-Castineira, F. Gonzalez-Castano, R. Duro, and F. LopezPena, "Urban pollution monitoring throught opportunistic mobile sensor networks based on public transport", Proceedings of the IEEE International Conference on Computational
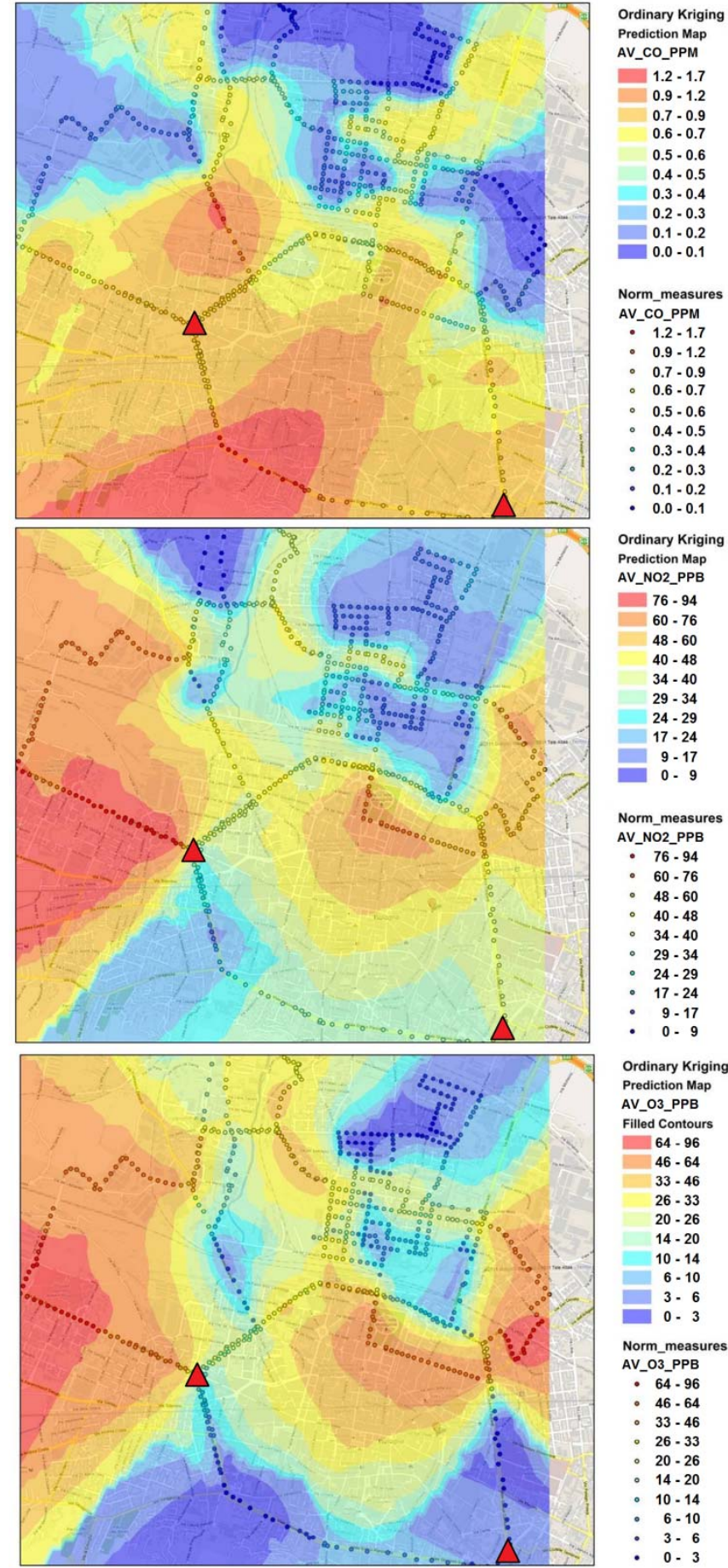

- $0-3$

Figure 8. Kriging variograms of the considered pollutants (the red triangles indicate the position of traditional fixed measurement systems).

Intelligence for Measurement Systems and Applications, 2008.

[5] Weber, C., Hirsch, J., Perron, G., Kleinpeter, J., Ranchin, T., Ung, A. and Wald, L., "Urban Morphology, Remote Sensing and Pollutants Distribution: An Application To The City of Strasbourg, France", International Union of Air Pollution Prevention and Environmental Protection Associations (IUAPPA) Symposium and Korean Society for Atmospheric Environment (KOSAE) Symposium, 12th World Clean Air \& Environment Congress, Greening the New Millennium, 26 - 31 August 2001, Seoul, Korea.

[6] G. Varela, A. Paz-Lopez, R. J. Duro, F. Lopez-Pena1, F. J. Gonzalez-Castano, "An Integrated System for Urban Pollution Monitoring through a Public Transportation based Opportunistic Mobile Sensor Network", IEEE International Workshop on 
Intelligent Data Acquisition and Advanced Computing Systems: Technology and Applications, 21-23 September 2009, Rende (Cosenza), Italy.

[7] V. Cavalho,J.G. Lopes, H.G. Ramos, F. Correa Alegria, "CityWide Mobile Air Quality Measurement System", Proceedings of IEEE Sensors 2009 Conference, Christchurch (NZL), 25-28 Oct. 2009, pp. 546-551.

[8] G. Betta, D. Capriglione, L. Ferrigno, G. Miele, "Influence of Wifi Computer Interfaces On Measurement Apparatuses", IEEE Transactions On Instrumentation And Measurement. vol. 59, Issue 12, Dec 2010, pp. 3244-3252.

[9] Ferrigno L., Paciello V.,Pietrosanto A., "Low-cost visual sensor node for BlueTooth-based measurement networks," IEEE Transactions on Instrumentation and Measurement, vol. 55, no.2, April 2006, pp. 521- 527.

[10] G. Betta, D. Capriglione, L. Ferrigno, G. Miele, "Experimental Investigation of the Electromagnetic Interference of Zigbee Transmitters on Measurement Instruments", IEEE Transactions On Instrumentation and Measurement. Vol.57, Issue 10, Oct 2008, pp. 2118-2127.

[11] A. Bernieri, D. Capriglione, L. Ferrigno, M. Laracca, "A mobile measurement system for urban pollution monitoring", Proceedings of the XIII TC-4 IMEKO Symposium and IX Semetro, Natal, Brazil, September 2011.
[12] Directive 1999/30/EC of 22 April 1999 relating to limit values for sulphur dioxide, nitrogen dioxide and oxides of nitrogen, particulate matter and lead in ambient air.

[13] Directive 2000/69/EC of the European Parliament and of the Council of 16 November 2000 relating to limit values for benzene and carbon monoxide in ambient air.

[14] [online] http://www.kanomaxusa.com/iaq/Sensor/Gas_sensor_head.html.

[15] [online] http://www.advantech.com.

[16] B. Denby, V. Garcia, F. Holland, C. Hogrefe, "Integration of air quality modeling and monitoring for enhanced health exposure assessment", Special Edition of EM Magazine "Monitoring and Modeling Needs in the $21^{\text {st }}$ Century".

[17] B. Denby, J. Horálek, S.E. Walker, K. Eben, and J. Fiala, "Interpolation and assimilation methods for European scale air quality assessment and mapping, Part I: Review and recommendations, ETC/ACC Technical Paper 2005/7.

[18] J. Horálek, P. Kurfürst, B. Denby, P. de Smet, F. de Leeuw, M. Brabec, J. Fiala, "Interpolation and assimilation methods for European scale air quality assessment and mapping, Part II: Development and testing new methodologies", ETC/ACC Technical Paper 2005/7. 